\title{
Aposematism in pink warty sea cucumbers: independent effects of chromatic and achromatic cues
}

\author{
Amanda Y. H. Lim*, Ian Z. W. Chan*,**, Luis R. Carrasco, Peter A. Todd \\ Department of Biological Sciences, National University of Singapore, 14 Science Drive 4, Singapore 117557
}

\begin{abstract}
The importance of chromatic and achromatic cues in aposematism is well established, but in situ experiments investigating their comparative roles within a single warning signal remain rare. In a predation experiment, we examined their relative importance in pink warty sea cucumbers Cercodemas anceps Selenka, 1867, by asking (1) Do chromatic and achromatic cues have significant effects on attack rate? (2) Are their effects independent? (3) Does one have more influence on attack rate than the other? Using a multispectral imaging-based technique, we fabricated 4 types of clay models based on the hues, chroma and luminance levels of the animals and the background. Models were deployed in sets of 4 at 2 sites $(n=65$ sets) for 3 days, and the number of imprints from predation attempts was recorded. A generalised linear mixed model analysis showed that both types of cues had significant but independent effects on attack rates and that chromatic cues had a greater effect compared to achromatic ones. This study, the first manipulative investigation into holothurian aposematism, demonstrates the potential for chromatic and achromatic cues to play distinct roles in animal signalling, and highlights the importance of accurate experimental models.
\end{abstract}

KEY WORDS: Warning colouration - Holothurian - Marine predation - Conspicuousness · Multispectral imaging $\cdot$ Cercodemas anceps

\section{INTRODUCTION}

Aposematism, the association between warning signals and prey unprofitability, is a well known phenomenon with a long history of study, especially in terrestrial environments (e.g. Brodie 1993, Prudic et al. 2007). However, less is known about aposematism in marine species. Only opisthobranchs have been studied (e.g. Ritson-Williams \& Paul 2007), while in other marine species with both conspicuous colouration and chemical defences, aposematism is generally assumed without experimental verificationsince this claim was made by Rudman (1991), only a handful of studies on nudibranch aposematism have been published (e.g. Aguado \& Marin 2007). A key element of aposematic signalling is conspicuousness, which improves avoidance learning (Roper \& Wistow

*These authors contributed equally to this work

** Corresponding author: ianchan@nus.edu.sg
1986) by naïve predators, and reduces recognition errors (Guilford 1986) and increases distinctiveness (Merilaita \& Ruxton 2007) for experienced predators. Conspicuousness, however, is itself poorly defined in the literature (Harvey \& Paxton 1981) and is often loosely used to refer to multiple related yet distinct properties of an animal's appearance, which comprises both chromatic and achromatic cues (Osorio \& Vorobyev 2005).

Chromatic cues consist of hue (or spectral position, which is perceived as differences in light spectra) and chroma (or spectral purity; Osorio \& Vorobyev 2005). The achromatic cue in colour is luminance, also often referred to as apparent lightness or brightness, which is a measure of intensity or light reflectance (Osorio \& Vorobyev 2005). Numerous previous studies have shown that animals with hues

() The authors 2019. Open Access under Creative Commons by Attribution Licence. Use, distribution and reproduction are unrestricted. Authors and original publication must be credited. 
or chroma levels not common in their environment display strong chromatic contrast against the background, contributing to recognition and avoidance for experienced predators, e.g. domestic chickens Gallus gallus domesticus preying on larvae of the seed bug Tropidothorax leucopterus (GamberaleStille 2001). Similarly, luminance contrast plays a critical role in signal recognition of simple monochromatic lepidopteran eyespots by experienced avian predators (Stevens et al. 2008). Signal learning and memory can also be influenced by hue itself irrespective of chromatic contrast (Harvey \& Paxton 1981) or by high luminance contrast against the background, e.g. in predators such as great tits Parus major (Sandre et al. 2010) and the colour-blind Chinese mantid Tenodera aridifolia sinensis (Prudic et al. 2007). Predators have also been shown to innately avoid certain hues (Miller \& Pawlik 2013) or high luminance in the form of specular reflectance (glossiness) (Waldron et al. 2017).

Although it is well known that animals use chromatic and achromatic cues for different purposes (Osorio \& Vorobyev 2005), few studies have attempted to tease apart their individual roles in the efficacy of the same signal (Sandre et al. 2010, Skelhorn et al. 2016). For example, while Prudic et al. (2007) suggested that both types of cues could together increase the efficiency of aposematic colouration for predators with colour vision, this interaction has yet to be tested. Psychophysics-based studies (e.g. Osorio \& Vorobyev 2005) have shown the perception of chromatic and achromatic cues to be distinct in animal visual systems, as comprehensively reviewed by Stevens (2007), giving rise to questions such as whether one may be more important than the other, whether their relative importance may change in different situations, and whether their effects interact in an additive or synergistic manner. In order to create a more complete understanding of how animals communicate information to one another visually, these questions need to be answered, and it is hence necessary for studies to examine the effects of chromatic and achromatic cues separately. However, doing so has thus far proven difficult to achieve. One reason for this knowledge gap is the difficulty of reliably manipulating specific aspects of an organism's appearance whilst keeping the other constant. This is an issue that we address here.

One marine taxon containing conspicuously coloured, unpalatable species are the holothurians. For example, the pink warty sea cucumber Cercodemas anceps Selenka, 1867 (Fig. 1a), from the family Cucumariidae, has a bright pink and yellow coloura- tion that appears conspicuous in its natural environment, the tropical intertidal seagrass meadows ranging from southern China to northern Australia. Like most other holothurians, this species also possesses a chemical defence in the form of cytotoxic triterpene saponins (Cuong et al. 2015), which suggests an aposematic function for its conspicuous colouration. However, the protective value of its appearance has not been confirmed experimentally. Aposematic signals can be effective in marine environments (e.g. Ritson-Williams \& Paul 2007), but signal efficacy may differ in the aquatic medium compared to more wellstudied terrestrial examples. For example, water itself causes patchy lighting conditions (and may thereby affect achromatic cues) and disproportionately attenuates light of longer wavelengths (red and infrared) with increasing depth (affecting chromatic cues) (Pegau et al. 1997), and the nature and concentration of suspended solids and organic matter within the water column also alter the ambient light field (Gallegos \& Moore 2000). Hence, it would be useful to demonstrate whether the appearance of $C$. anceps is indeed aposematic.

Our study aimed to investigate whether the appearance of $C$. anceps has an aposematic function, and if so, to disentangle the relative importance of chromatic and achromatic cues. We conducted a fieldbased predation experiment using modelling clay replicas of $C$. anceps, which were fabricated to match target hues, chroma and luminance levels using a robust procedure, and asked 3 questions: (1) Do chromatic and achromatic cues have significant effects on attack rate? (2) Are their effects independent? (3) Does one have more influence on attack rate than the other? We hypothesised that both types of cues would
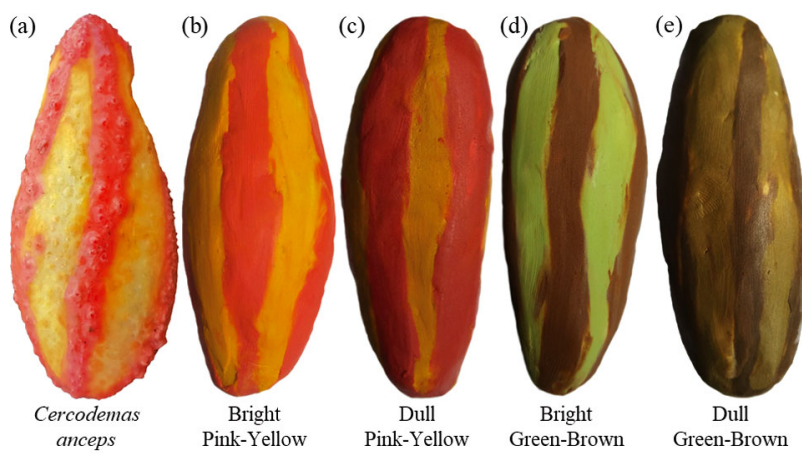

Fig. 1. (a) Live pink warty sea cucumber, (b) bright pink-yellow model (with similar hues, chroma and luminance to the actual animal), (c) dull pink-yellow model (with similar hues and chroma but lower luminance), (d) bright green-brown model (with different hues and chroma but similar luminance) and (e) dull green-brown model (with different hues and chroma and lower luminance) 
have significant, interacting effects but that chromatic cues would have a greater protective effect.

\section{MATERIALS AND METHODS}

\subsection{Model fabrication}

Four types of models were fabricated using nontoxic modelling clay (Sargent Art Modeling Clay Plastilina, in 'Cream') and Americolor Soft Gel Paste food colouring to display chromatic cues (i.e. hues and chroma levels) and achromatic cues (i.e. luminance levels) that were either conspicuous (i.e. similar to living Cercodemas anceps) or non-conspicuous (i.e. similar to the natural background). The colour combinations used were bright pink-yellow (with conspicuous luminance and hues and chroma), bright brown-green (with conspicuous luminance but nonconspicuous hues and chroma), dull pink-yellow (with non-conspicuous luminance but conspicuous hues and chroma) and dull brown-green (with nonconspicuous luminance and hues and chroma) (Fig. 1b-e). The brown and green hues used were chosen because they are common and not typically associated with aposematic colouration in the natural habitat of the study species, an approach employed in predation studies, e.g. on coral snakes Micrurus sp. (Brodie 1993).

To establish the target hue, chroma and luminance levels, 35 sea cucumbers and their backgrounds were digitally photographed at Changi Beach, Singapore, and analysed. All images were captured as RAW files using a full-spectrum Nikon D7100 body with a Jenoptik CoastalOpt 105 mm UV-Vis crystal lens and Baader UV/IR Cut filter, and included 3\% and $97 \%$ grey reflectance standards (made by Jolyon Troscianko). The Multispectral Image Calibration and Analysis (MICA) Toolbox (Troscianko \& Stevens 2015) in ImageJ was used to correct for different lighting conditions and irregular camera spectral response, and to convert the RAW images into linearised, objective (i.e. camera independent) redgreen-blue (RGB) pixel values. Finally, pixel values were sampled using the random sampler tool in PATGEOM (Chan et al. 2019), which calculates the average RGB values of 30 randomly selected $10 \times 10$ square pixel portions of the regions of interest, i.e. green and brown areas of the seagrass background, and pink and yellow areas of the sea cucumbers.

Luminance was matched using the normalised sum of the RGB values (Troscianko \& Stevens 2015), hue was measured using R:G, R:B and G:B ratios, and chroma (also known as saturation) was calculated following Agoston (2005):

$$
\text { Chroma }=\left\{\begin{array}{lr}
0, & \text { if } L=1 \\
\frac{\max (R, G, B)-\min (R, G, B)}{1-|2 L-1|}, & \text { otherwise }
\end{array}\right.
$$

where: $\max (R, G, B)$ is the largest of the $R, G$ or $B$ values; $\min (R, G, B)$ is the smallest of the $R, G$ or $B$ values; and $L=[\max (\mathrm{R}, \mathrm{G}, \mathrm{B})+\min (\mathrm{R}, \mathrm{G}, \mathrm{B})] / 2$.

Objective RGB values provide an accurate representation of appearance as they measure the actual intensity of the light coming from an object (Troscianko \& Stevens 2015). They were not converted to predator visual spaces because (1) sea cucumbers are preyed upon by more than one type of predator, and (2) if the RGB values of the models are designed to match target RGB values measured from either the sea cucumbers (for conspicuous cues) or the substrate (for non-conspicuous cues), they should display the desired conspicuous or non-conspicuous chromatic and achromatic cues to predators. Clay swatches were then created, analysed in the same manner, compared to their target colours in actual C. anceps and the seagrass background, and iteratively adjusted by varying the amount of colouring used until they fell within the observed ranges of their target hue and chroma and luminance levels (Fig. S1 in Supplement 1 at www.int-res. com/articles/suppl/m631p157_supp/; the final amounts and types of food colouring used for each colour are provided in Table S1 in Supplement 1). Finally, each model was fabricated using $40 \mathrm{~g}$ of appropriately-coloured clay moulded to a length of $7 \mathrm{~cm}$ (based on the lengths of 30 individuals measured prior to the experiment).

\subsection{Predation experiment}

The predation experiment was performed at 2 intertidal seagrass meadows in Singapore where C. anceps has been observed: Changi Beach (on 16 November 2016) and Tanah Merah (on 10 January 2017). Models were mounted on L-shaped aluminium stakes that were driven into the muddy substrate so that no part of the stakes was exposed. The models were deployed in sets of 4 arranged in a square of side length $50 \mathrm{~cm}$, with 1 model of each type positioned randomly at each corner of the square. Sets were spaced at least $5 \mathrm{~m}$ apart. A total of 65 sets (32 at Changi and 33 at Tanah Merah) were left in the field for 3 days, after which they were collected and the number of imprints from predation attempts on each model was recorded. The models were emersed for approximately $6 \mathrm{~h}$ total per day (over 2 low tides) and for the 
remaining time submerged to a maximum of $3 \mathrm{~m}$, sufficiently shallow that ambient light during the day would still contain most of the original (i.e. abovewater) intensity in all of the visible wavelengths. The density of the deployed models was generally lower than natural sea cucumber densities at the sites and was chosen to minimise the possibility of the same predator encountering multiple sets.

For imprints on the models to be considered a predation attempt, they had to be at least $1 \mathrm{~mm}$ deep, and form regularly spaced ridges or indentations (for bite imprints) or the modelling clay had to have been gouged, pinched or pushed aside by force (for claw imprints) (Fig. 2). Imprints were counted as a single predation attempt when found in clear pairs, i.e. when both imprints had similar depth, length and curvature, but mirrored, such as might be made by crab chelae. The number of bites on each model was analysed using a generalised linear mixed model (GLMM) with site and set as random effects (which also accounts for potentially different environmental conditions and predator communities at the 2 sites on the 2 deployment dates), and using a negative binomial error distribution to account for over-dispersion. Another GLMM was also performed on whether each clay model was attacked (with a binomial error distribution and the same fixed and random effects). As the results of the 2 analyses were very similar (Tables $\mathrm{S} 2 \& \mathrm{~S} 3$ in Supplement 1) and the former is able to take into account information on both whether and how many times each model was attacked (which is useful if multiple predators visited a set), only the results of the analysis on number of bites are presented. Models were also checked to ensure there were no issues with heteroscedasticity or normality of errors.
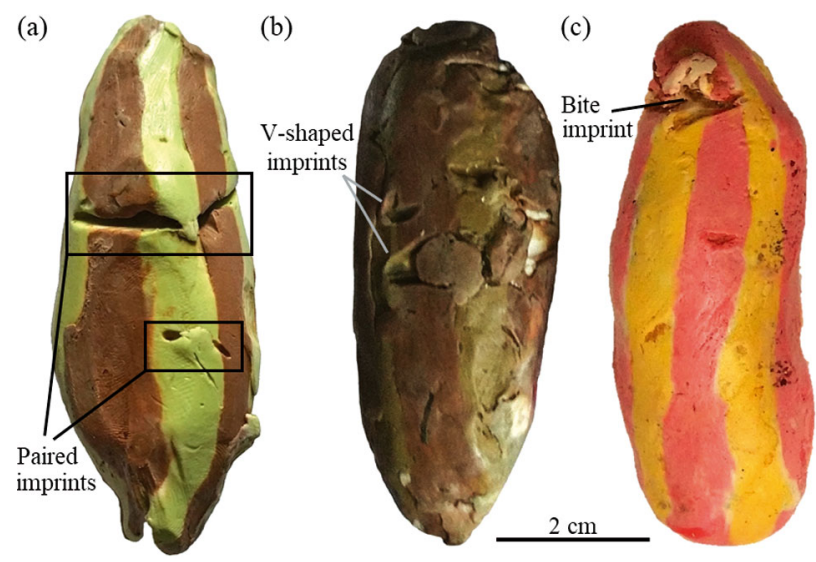

Fig. 2. Examples of predation imprints found on the models: (a) paired imprints likely made by crab chelae, (b) V-shaped imprints characteristic of marks left by avian beaks and (c) bite mark likely made by a fish
Statistical analyses were conducted using the 'lme4' package (Bates et al. 2015) in R (version 3.4.3) (R Core Team 2017).

\section{RESULTS}

All 260 models were recovered and 215 exhibited imprints from predation attempts. Examples include deep paired imprints (which were the most common), large bite marks, rows of small teeth-shaped impressions and V-shaped imprints (Fig. 2). We do not have video evidence of the predators and therefore cannot confirm which animals are responsible for each type of imprint. However, the V-shaped imprints (Fig. 2b) are similar to avian beak marks photographed in other studies (e.g. Howe et al. 2009), and birds are known to consume sea cucumbers (Francour 1997). It is also possible that the paired imprints (Fig. 2a) and bite marks (Fig. 2c) were made by crab chelae and fish bites, a suggestion which is consistent with both existing knowledge and our field observations: crabs and fish are known predators of sea cucumbers (Francour 1997) and have been seen to prey on them at our sites (authors' pers. obs.). However, we were unable to find photographic records of crab chelae marks and fish bites on clay models due to the relatively few aquatic predation studies using this technique. This is therefore speculative, and further research would be needed to conclusively identify the predators attacking the clay models.

Bright pink-yellow models were the least attacked, with the smallest mean number of imprints per model (mean \pm SE: $1.44 \pm 0.18$ imprints) and proportion of models attacked ( 39 of 65 , or $60 \%$ ). Dull brown-green models, conversely, were the most attacked $(4.71 \pm$ $0.58 ; 100 \%$ of models attacked). This suggests that the pink-yellow colouration did have a protective effect. The other 2 model types experienced intermediate levels of attack, with dull pink-yellow models attacked less $(2.28 \pm 0.28 ; 76.9 \%)$ than the bright brown-green models $(3.82 \pm 0.47 ; 93.8 \%)$, suggesting that the chromatic cue has a greater protective effect (Fig. 3a). The GLMM confirms these results: both achromatic $(p<0.001)$ and chromatic cues $(p<1.0 \times$ $10^{-15}$ ) have significant effects on the number of predator imprints per model, which are independent (the interaction between the two is not significant, $\mathrm{p}=$ 0.26) (Table 1, Fig. 3b). In a second model, without the non-significant interaction term (Table 2; Fig. 3c), it is clear that the chromatic cue (effect size: $\beta=0.842$ $\pm 0.097 \mathrm{SE}$ ) has a significantly greater effect on attack rates than the achromatic one $(\beta=0.307 \pm 0.094)$. 


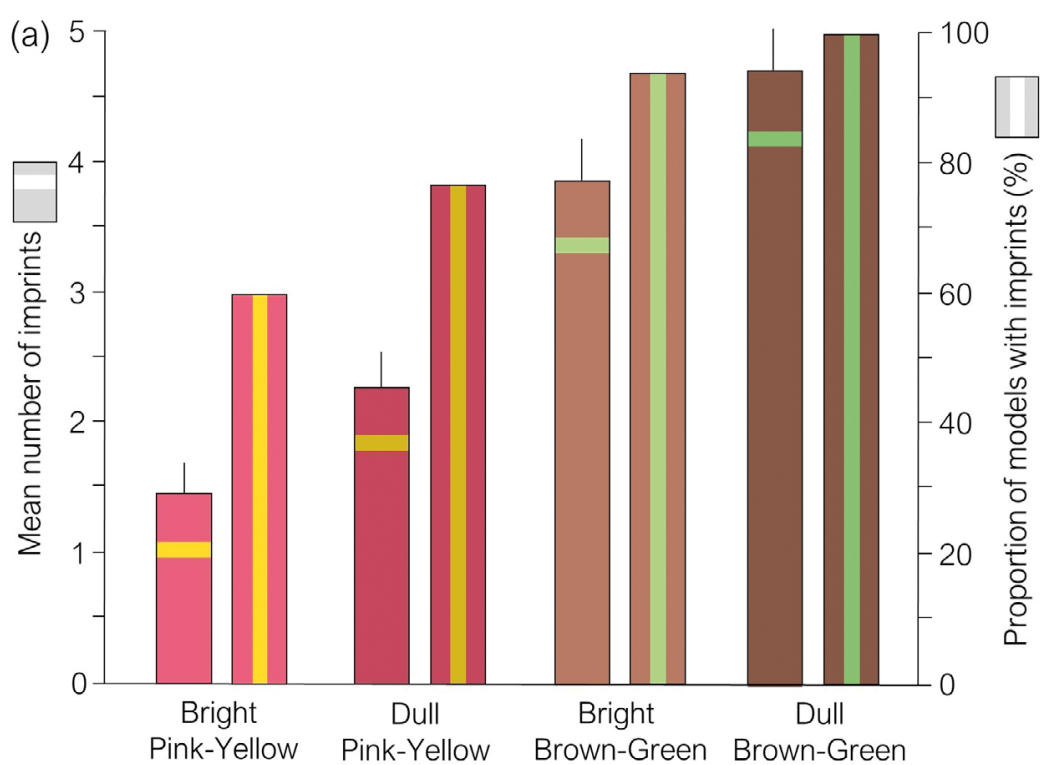

(b)

(c)
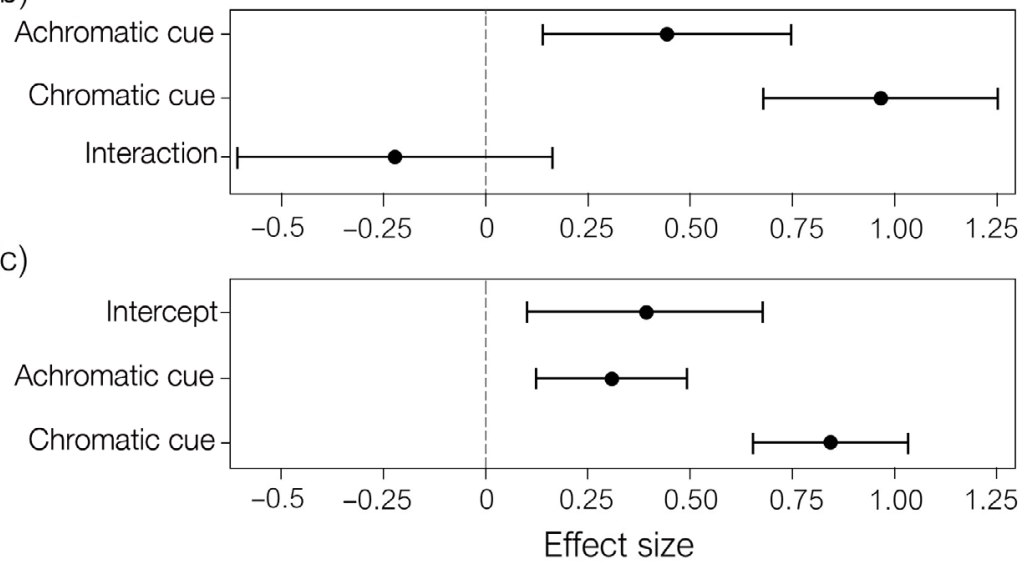

Fig. 3. (a) Mean (+SE) number of predation imprints observed on models (left axis) and proportion of models attacked (right axis) for each of the 4 model types. (b) Effect sizes of the chromatic cues, achromatic cues and their interaction, from a GLMM analysing their effects on the mean number of imprints on models. (c) Same as in (b) but without the interaction term (as it was nonsignificant). The bright pink-yellow models were designated as the reference (the Intercept) to which the other 3 types of models were compared. Ranges in

(b) and (c) represent $95 \%$ confidence intervals.

Table 1. Generalised linear mixed model (GLMM) results of the full model show significant and independent effects for the chromatic and achromatic cues on attack rates; ${ }^{*} \mathrm{p}<0.05 ;{ }^{* *} \mathrm{p}<0.001$

\begin{tabular}{|lcccc|}
\hline Variable & $\beta$ & SE & $Z$ & $p$ \\
\hline $\begin{array}{l}\text { Intercept } \\
\begin{array}{c}\text { Achromatic cue } \\
\text { (dull) }\end{array}\end{array}$ & 0.312 & 0.135 & 2.306 & $0.021^{*}$ \\
$\begin{array}{c}\text { Chromatic cue } \\
\text { (brown-green) }\end{array}$ & 0.965 & 0.147 & 6.579 & $<0.0001^{* *}$ \\
Interaction & -0.221 & 0.196 & -1.128 & 0.259 \\
\hline
\end{tabular}

\section{DISCUSSION}

The importance of conspicuousness in aposematism is widely accepted, but in situ experimental evidence remains rare, and the relative roles of chromatic and achromatic cues within a single signal are seldom examined. Using a quantitatively robust process to fabricate precise models of pink warty sea cucumbers Cercodemas anceps, we conducted predation experiments to investigate whether the sea cucumbers' colour patterns serve an aposematic function and tested the effects of chromatic and achromatic cues. Fewer predation attempts were observed on realistic (bright pinkyellow) models compared to those with a less conspicuous (dull greenbrown) appearance, indicating that the appearance of $C$. anceps had a protective function. In addition, we demonstrated that the effects of both types of cues were significant and independent of each other, and that chromatic cues had a greater effect on attack rate. To our knowledge, this study represents the first attempt to disentangle the relative importance of chromatic and achromatic cues in aposematic colouration within a single manipulative experiment.

While the predation rates observed in our study are high $(96 \%$ of bright brown-green models and all dull brown-green models exhibited predation imprints), animals within seagrass beds (where we deployed our models) are known to experience moderately high predation pressure (Orth et al. 1984) and this may be augmented by

Table 2. GLMM results of the second model (without the interaction term) show that the chromatic cue was more influential than the achromatic cue; ${ }^{*} \mathrm{p}<0.01 ;{ }^{* *} \mathrm{p}<0.001$

\begin{tabular}{|lcccl|}
\hline Variable & $\beta$ & SE & $Z$ & \multicolumn{1}{c|}{$p$} \\
\hline $\begin{array}{l}\text { Intercept } \\
\text { Achromatic cue }\end{array}$ & 0.391 & 0.114 & 3.425 & $<0.001^{* *}$ \\
$\begin{array}{c}\text { (dull) } \\
\text { Chromatic cue } \\
\text { (brown-green) }\end{array}$ & 0.842 & 0.094 & 3.260 & $<0.01^{*}$ \\
\hline
\end{tabular}


the movement of organisms, including predators, amongst the various types of marine habitats that can be found in Singapore, a tropical island state with seagrass beds, rocky and sandy shores, coral reefs and mangrove forests (Grober-Dunsmore et al. 2009). Animal taxa that consume holothurians include fish, crabs and, to a lesser extent, birds (Francour 1997) all of which are potentially responsible for predation attempts in the current experiment (although we were unable to confirm this with video evidence) and are known to possess colour vision (Bennett \& Théry 2007, Detto 2007, Bowmaker 2008). Birds are the most common predators in experimental studies of aposematism, reef fishes have been used to test aposematism in opisthobranchs (e.g. Aguado \& Marin 2007), and crabs have also been observed responding to warning signals (Grober 1988).

If these taxa - which are highly mobile, e.g. fish $>20 \mathrm{~cm}$ in length (which are present at our study sites) can have home ranges of $0.1-10 \mathrm{~km}^{2}$ (Kramer \& Chapman 1999), and birds that frequent both sites (such as common mynahs Acridotheres tristis, weighing 110-140 g) typically possess home ranges of between 4000 and $40000 \mathrm{~m}^{2}$ (Schoener 1968) - are indeed responsible for the attacks on our models, it is possible that the same predator may have encountered more than one set of models. Nevertheless, the wide variety of different types of imprints of different sizes we observed suggests that repeat visits, if present, represent at most a small portion of our data. Furthermore, the large difference in the proportion of bright pink-yellow models (60\%) compared to dull brown-green models attacked (100\%) remains a clear indication that the appearance of the former does confer some protection from predators, i.e. it is aposematic. In demonstrating aposematism in a holothurian, our study extends previous research into warning colouration in marine environments beyond the opisthobranchs and provides further evidence that marine predators do possess the ability to recognise aposematic signals.

It should be noted that an unavoidable consequence of our experimental design is that the 4 models within any given set are not independent of one another, i.e. each attack decision is a choice among them. However, we were able to account for this by including a set identifier number as a random effect in the data analysis. Furthermore, this design ensured that predators encountered each model type equally and within similar environmental conditions (e.g. ambient light and turbidity), and still gives an accurate indication of which model or models are avoided more than others. It also at least partially addresses a common problem in studies on aposematism (e.g. Brodie 1993): the controls in such experiments are typically of a similar colour to the background substrate and may benefit from background matching. This makes it difficult to measure how much protection is conferred by the warning colouration being studied. Within our sets, however, the models with high chromatic and/or achromatic contrast would likely draw a predator's attention to both themselves and to the more cryptic models nearby.

Comparing $C$. anceps models of similar luminance, those with pink and yellow hues experienced significantly fewer attacks compared to those with brown and green hues. This was the case both for bright models (with luminance similar to those of wild $C$. anceps) and for dull models (those similar to the background luminance), suggesting that predators separated chromatic and achromatic cues in natural conditions, and agrees with existing laboratory studies on the role of hue in predator memory and recognition of signals (e.g. Guilford 1986, Osorio et al. 1999; but luminance is not always considered in these studies). It is possible that the brown and green striping on two of the models provided a degree of camouflage or elicited novel stimuli predator reactions (i.e. neophobia or dietary conservatism; Marples \& Kelly 1999). Any of these processes, if in effect, would reduce attack rates. Hence, the aposematic effects of the pink and yellow (which occur naturally in the environment and would not cause neophobia) may actually be stronger than reported here. Although less influential than the chromatic cue in the GLMM, the achromatic cue, luminance, was still important: within both colour types, bright models were attacked less than dull ones. This lends support to the work of Prudic et al. (2007) and Sandre et al. (2010) showing that luminance has an effect on predator responses to prey models. It must be noted that predators in those experiments were naïve, whereas we assumed that the ones in the current study were not, and therefore different processes were likely involved (signal learning vs. prey recognition). In addition, our results do not separate the effects of chromatic or achromatic cues from aposematism but try instead to determine which is more important to experienced predators in recognising the aposematic signal displayed by C. anceps. The greater influence of the chromatic cue in this experiment suggests that while both types of cues were important, its predators relied more on chromatic cues under natural conditions.

Prudic et al. (2007) proposed that chromatic contrast and luminance contrast in combination would 
increase the effectiveness of aposematic colouration. This is what we found, as the model with the highest luminance and pink-and-yellow hues (i.e. the one most resembling $C$. anceps) was the least attacked by predators. Since we did not find an interaction, this increase was additive. Such a presence of additive, but not synergistic, effects also agrees with the predictions of Rowe (1999) regarding signals whose components are transmitted through the same sensory modality (in this case, vision). There are indications that chromatic and achromatic cues play different roles in signal recognition, e.g. small objects and textures are primarily discriminated by luminance (Newport et al. 2017), whereas hue is more important for the identification and discrimination of large objects (Osorio et al. 1999). Alternatively, these 2 signal components may simply have the same function in order to better communicate with a variety of predators with differing sensitivities to either type of cue, thus broadening the range of receivers for the warning signal (Ratcliffe \& Nydam 2008). Although chromatic and achromatic contrast had qualitatively similar effects in this instance, this may not necessarily be the case in other systems. This uncertainty highlights the importance of developing accurate model fabrication techniques to broaden these results, e.g. by attempting to separate the roles of hue and chroma in signalling in a variety of animals with different visual systems, life histories and environmental contexts. The ability demonstrated here to accurately control the individual visual properties of the artificial models used in predation experiments is crucial for researchers attempting to tease apart the effects of chromatic and achromatic cues and better understand predator learning and cognition (Skelhorn et al. 2016).

Our results, however, also raise an interesting question: if conspicuous hues, chroma and luminance levels do indeed protect unpalatable organisms from predation, why is it that not all apparently distasteful holothurians appear aposematic? For opisthobranchs, Tullrot (1994) suggested that cryptic colouration and unpalatability are able to coexist as unrelated defence mechanisms because their chemical defences are diet-derived and thus low-cost. However, this is unlikely to be the case in holothurians, as their chemical defences are synthesised de novo (Kerr \& Chen 1995) and thus incur a comparatively higher cost. It may be that non-aposematic holothurians are protected by other characteristics such as crypsis or burrowing behaviour (Bakus 1973), reducing the benefits of conspicuous colouration. Variation among predator species in dietary conser- vatism, prey handling and susceptibility to chemical defences could also impact the effectiveness of aposematism in different prey species (Endler \& Mappes 2004). Since many holothurians have similar chemical defences but very variable appearances, they are a promising taxon for investigating the evolution of aposematism.

Acknowledgements. We thank Aaron Teo for his help with the statistical analysis as well as the other members of the Experimental Marine Ecology Lab for their advice and support. Singapore National Parks Board kindly provided the permit for this experiment (NP/RP16-138). This work was supported by the Singapore Ministry of Education (grant number R-154-000-660-112). All applicable international, national and/or institutional guidelines for the care and use of animals were followed. All data and the R code used for the GLMMs are included as Supplement $2 \& 3$.

\section{LITERATURE CITED}

Agoston MK (2005) Computer graphics and geometric modeling: implementation and algorithms. Springer, London

Aguado F, Marin A (2007) Warning coloration associated with nematocyst-based defences in aeolidiodean nudibranchs. J Molluscan Stud 73:23-28

* Bakus GJ (1973) Biology and ecology of tropical holothurians. In: Jones OA, Endean R (eds) Biology and geology of coral reefs. Academic Press, New York, NY, p 325-367

* Bates D, Mächler M, Bolker B, Walker S (2015) Fitting linear mixed-effects models using lme4. J Stat Softw 67:1-48

* Bennett ATD, Théry M (2007) Avian color vision and coloration: multidisciplinary evolutionary biology. Am Nat 169:S1-S6

Bowmaker JK (2008) Evolution of vertebrate visual pigments. Vision Res 48:2022-2041

* Brodie ED III (1993) Differential avoidance of coral snake banded patterns by free-ranging avian predators in Costa Rica. Evolution 47:227-235

Chan IZW, Stevens M, Todd PA (2019) PAT-GEOM: a software package for the analysis of animal patterns. Methods Ecol Evol 10:591-600

* Cuong NX, Le TV, Tran THH, Nguyen PT and others (2015) Cytotoxic triterpene saponins from Cercodemas anceps. Bioorg Med Chem Lett 25:3151-3156

* Detto T (2007) The fiddler crab Uca mjoebergi uses colour vision in mate choice. Proc R Soc B 274:2785-2790

Endler JA, Mappes J (2004) Predator mixes and the conspicuousness of aposematic signals. Am Nat 163:532-547

* Francour P (1997) Predation on holothurians: a literature review. Invertebr Biol 116:52-60

Gallegos CL, Moore KA (2000) Factors contributing to water-column light attenuation. In: Batiuk RA (ed) Chesapeake Bay submerged aquatic vegetation water quality and habitat-based requirements and restoration targets: a second technical synthesis. United States Environmental Protection Agency, Annapolis, MD, p 35-54

Gamberale-Stille G (2001) Benefit by contrast: an experiment with live aposematic prey. Behav Ecol 12: $768-772$ 
Grober MS (1988) Brittle-star bioluminescence functions as an aposematic signal to deter crustacean predators. Anim Behav 36:493-501

Grober-Dunsmore R, Pittman SJ, Caldow C, Kendall MS, Frazer TK (2009) A landscape ecology approach for the study of ecological connectivity across tropical marine seascapes. In: Nagelkerken I (ed) Ecological connectivity among tropical coastal ecosystems. Springer Netherlands, Dordrecht, p 493-530

Guilford T (1986) How do 'warning colours' work? Conspicuousness may reduce recognition errors in experienced predators. Anim Behav 34:286-288

Harvey PH, Paxton RJ (1981) The evolution of aposematic coloration. Oikos 37:391-396

Howe A, Lövei GL, Nachman G (2009) Dummy caterpillars as a simple method to assess predation rates on invertebrates in a tropical agroecosystem. Entomol Exp Appl 131:325-329

Kerr RG, Chen Z (1995) In vivo and in vitro biosynthesis of saponins in sea cucumbers. J Nat Prod 58:172-176

Kramer DL, Chapman MR (1999) Implications of fish home range size and relocation for marine reserve function. Environ Biol Fishes 55:65-79

Marples NM, Kelly DJ (1999) Neophobia and dietary conservatism: two distinct processes? Evol Ecol 13:641-653

Merilaita S, Ruxton GD (2007) Aposematic signals and the relationship between conspicuousness and distinctiveness. J Theor Biol 245:268-277

Miller AM, Pawlik JR (2013) Do coral reef fish learn to avoid unpalatable prey using visual cues? Anim Behav 85: 339-347

Newport C, Green NF, McClure EC, Osorio DC, Vorobyev M, Marshall NJ, Cheney KL (2017) Fish use colour to learn compound visual signals. Anim Behav 125:93-100

Orth RJ, Heck KL, van Montfrans J (1984) Faunal communities in seagrass beds: a review of the influence of plant structure and prey characteristics on predator-prey relationships. Estuaries 7:339-350

Osorio D, Vorobyev M (2005) Photoreceptor spectral sensitivities in terrestrial animals: adaptations for luminance and colour vision. Proc R Soc B 272:1745-1752

Osorio D, Jones CD, Vorobyev M (1999) Accurate memory for colour but not pattern contrast in chicks. Curr Biol 9: 199-202

Pegau WS, Gray D, Zaneveld JRV (1997) Absorption and attenuation of visible and near-infrared light in water:

Editorial responsibility: Antony Underwood, Sydney, New South Wales, Australia dependence on temperature and salinity. Appl Opt 36: 6035-6046

*Prudic KL, Skemp AK, Papaj DR (2007) Aposematic coloration, luminance contrast, and the benefits of conspicuousness. Behav Ecol 18:41-46

R Core Team (2017) R: a language and environment for statistical computing. R Foundation for Statistical Computing, Vienna

Ratcliffe JM, Nydam ML (2008) Multimodal warning signals for a multiple predator world. Nature 455:96-99

Ritson-Williams R, Paul VJ (2007) Marine benthic invertebrates use multimodal cues for defense against reef fish. Mar Ecol Prog Ser 340:29-39

Roper TJ, Wistow R (1986) Aposematic colouration and avoidance learning in chicks. Q J Exp Psychol B 38: 141-149

Rowe C (1999) Receiver psychology and the evolution of multicomponent signals. Anim Behav 58:921-931

Rudman WB (1991) Purpose in pattern: the evolution of colour in chromodorid nudibranchs. J Molluscan Stud 57: $5-21$

Kandre SL, Stevens M, Mappes J (2010) The effect of predator appetite, prey warning coloration and luminance on predator foraging decisions. Behaviour 147: 1121-1143

Schoener TW (1968) Sizes of feeding territories among birds. Ecology 49:123-141

Skelhorn J, Halpin CG, Rowe C (2016) Learning about aposematic prey. Behav Ecol 27:955-964

* Stevens M (2007) Predator perception and the interrelation between different forms of protective coloration. Proc R Soc B 274:1457-1464

Stevens M, Stubbins CL, Hardman CJ (2008) The antipredator function of 'eyespots' on camouflaged and conspicuous prey. Behav Ecol Sociobiol 62:1787-1793

* Troscianko J, Stevens M (2015) Image calibration and analysis toolbox - a free software suite for objectively measuring reflectance, colour and pattern. Methods Ecol Evol 6: 1320-1331

Tullrot A (1994) The evolution of unpalatability and warning coloration in soft-bodied marine invertebrates. Evolution 48:925-928

*Waldron SJ, Endler JA, Valkonen JK, Honma A, Dobler S, Mappes J (2017) Experimental evidence suggests that specular reflectance and glossy appearance help amplify warning signals. Sci Rep 7:257

Submitted: May 10, 2019 ; Accepted: October 7, 2019 Proofs received from author(s): November 18, 2019 